\title{
SÍNDROME DE BURNOUT EM RESIDENTES MÉDICOS DE MONTES CLAROS/MG
}

\author{
BURNOUT SYNDROME IN MEDICAL RESIDENTS FROM MONTES CLAROS/MG
}

\author{
Emillyo César Neves Ferreira ${ }^{a^{*}}$, Frederico Victor Ribeiro ${ }^{b^{*}}$, Rodrigo Pereira Santos Neto ${ }^{c^{*}}$, \\ Bruna Nathália Santos $^{\mathrm{d}^{* *}}$, Luçandra Ramos Espirito Santo ${ }^{\mathrm{e}^{* * *}}$, Karina Andrade Prince ${ }^{\mathrm{f}^{*}}$, Marcos \\ Vinícius Macedo de Oliveirag*

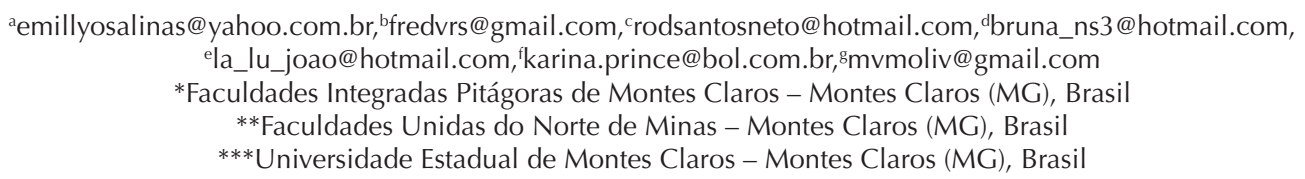

\section{RESUMO}

Introdução: A presença de fatores estressores e as condiçôes de trabalho dos médicos residentes favorecem o surgimento da síndrome de burnout, síndrome caracterizada por exaustão emocional, despersonalização e reduzida realização profissional. Objetivo: Verificar a existência de associação entre a síndrome de burnout e variáveis profissionais e sociodemográficas em médicos que integram os programas de residências médicas dos principais hospitais de Montes Claros/MG. Materiais e métodos: Utilizou-se como instrumento de pesquisa o questionário Maslach Burnout Inventory, juntamente com formulário de avaliação sociodemográfica. A amostra, aleatória e simples, foi composta por37 residentes de três hospitais de Montes Claros/MG. Resultados: Revelou-se alta ocorrência (75,7\%) de burnout entre os participantes, embora não tenha ficado evidente a relação com gênero, estado civil, renda familiar ou carga horária. Conclusáo: A despeito disso, que pode ter relação pela quantidade de residentes avaliados, os dados reforçam a importância de medidas de prevenção do desgaste físico e psíquico desses profissionais, como demonstrado pela elevada prevalência identificada neste estudo.

Palavras-chave: Residência médica; estresse; medicina.

\section{ABSTRACT}

Introduction: The presence of stressor factors and the work conditions of medical residents favor the emergence of burnout syndrome, characterized by emotional exhaustion, depersonalization and decrease of professional fulfilment. Objective: Verify the relation between the Burnout syndrome and professional and socio-demographic variables in doctors from the programs of medical residences of the main hospitals in Montes Claros/MG, Brazil. Materials and methods: The Maslach Burnout Inventory was used as a research tool, along with a socio-demographic assessment form. The sample consisted of 37 residents of three hospitals in Montes Claros/MG, Brazil. Results: A high rate (75.7\%) of burnout syndrome was shown among the participants, although it was not evident its relation with gender, marital status, family income or working hours. Conclusion: Nevertheless, the data reinforce the importance of preventive measures against the physical and mental exhaustion of these professionals, as demonstrated by the high prevalence identified in this study.

Keywords: Medical residency; stress; medicine. 


\section{Introdução}

Os programas de residência médica têm como objetivo proporcionar formaçáo de qualidade aos profissionais da saúde através de treinamento supervisionado em serviço, de forma que esses médicos, sendo estudantes e trabalhadores simultaneamente, passam a lidar com essa dualidade de funçôes ${ }^{1}$.

O período de duração da residência varia de dois a cinco anos de acordo com a especialidade, sob regime de 60horas semanais de atividades, sendo preconizada a realização de 80 a $90 \%$ da carga horária em forma de treinamento em serviço com supervisão médica qualificada e $10 \%$ a $20 \%$ por meio de atualizaçóes, seminários e correlação clinicopatológica, entre outras priorizadas pelos programas ${ }^{2}$. Nessa mesma época, esses profissionais convivem com situações como privação do sono, fadiga, excessiva carga assistencial, excesso de trabalho administrativo, problemas relativos à qualidade do ensino e ao ambiente educacional ${ }^{3}$.

A tradução do termo burnout significa "queima após desgaste". Fica claro nesse contexto, o emprego dessa nomenclatura para se referir a um quadro clínico bastante associado à ocorrência de exaustão de aspectos funcionais por desgaste excessivo, especialmente no contexto do trabalhador. A síndrome de burnout tipicamente se relaciona com a ocorrência da exaustão emocional, despersonalização e reduzida realização profissional. Essas características são mais propícias de existirem em trabalhadores que lidam usualmente com outras pessoas ${ }^{4}$.

Estudos realizados com profissionais e estudantes da área da saúde revelam a presença de fatores estressores e de condiçôes que favorecem o surgimento da síndrome de burnout (SB). Estudos em profissionais da saúde evidenciaram elevada presença dessa síndrome (de 50 a $74 \%$ ) entre professores médicos, grupos de enfermagem e residentes médicos, sendo que entre os estudantes de residência em clínica médica, notou-se uma prevalência de $63 \%$ da $\mathrm{SB}^{5-7}$. No Brasil, um estudo demonstrou a presença da SB em 78,4\% entre residentes de ortopedia, clínica médica, cirurgia, pediatria, ginecologia e obstetrícia em um hospital público da cidade de Uberlândia/ $\mathrm{MG}^{8}$.

O indivíduo com SB pode apresentar sintomas somáticos ou físicos (exaustão, fadiga crônica, cefaleias, distúrbios gastrintestinais, alteraçóes do sono, suscetibilidade a infecçôes, hipertensão), psicológicos (irritabilidade, ansiedade, baixa autoestima e desinteresse, apatia; e em níveis mais elevados, pode apresentar depressão, paranoia e atos suicidas), ou mesmo comportamentais (fazer consultas rápidas, evitar os pacientes e o contato visual, colocar rótulos depreciativos, usar tranquilizantes e/ou barbitúricos, aumentar o consumo de álcool, cafeína e/ou nicotina) ${ }^{9-12}$.
Este trabalho buscou identificar a ocorrência de síndrome de burnout em residentes médicos da rede hospitalar da cidade de Montes Claros/MG, analisando também algumas condiçốes sociodemográficas.

\section{Metodologia}

\section{Desenho do estudo}

Foi realizada uma pesquisa analítica, transversal, de natureza quantitativa, envolvendo 37 acadêmicos matriculados em residências médicas de hospitais da cidade de Montes Claros/MG no período entre março e junho de 2015. Os hospitais onde a pesquisa se desenvolveu foram: Santa Casa de Misericórdia de Montes Claros, Fundação Dilson de Quadros Godinho e Hospital Universitário Clemente de Faria. Para participação na pesquisa, era preciso estar matriculado nos cursos de residência médica há mais de seis meses e assinar o Termo de Consentimento Livre e Esclarecido. A coleta de dados ocorreu em ambiente com privacidade assegurada e em momento combinado com o participante, sempre na presença do pesquisador. Este estudo respeitou todos os preceitos éticos exigidos para pesquisas envolvendo seres humanos, mediante parecer favorável para sua execução segundo Comitê de Ética em Pesquisa (parecer: 887.367/2014).

O Hospital Santa Casa de Misericórdia de Montes Claros teve 9 estudantes investigados: nos cursos de residência em cirurgia plástica (1), cirurgia geral (2), ginecologia e obstetrícia (2), ortopedia e traumatologia (4). A Fundação Dilson de Quadros Godinho teve 10 estudantes investigados: nos cursos de residência em clínica médica (1), ortopedia e traumatologia (1), radioterapia (2), radiologia (6).

O Hospital Universitário Clemente de Faria teve 18 estudantes investigados: nos cursos de residência em anestesiologia (2), cirurgia geral (2), clínica médica (4), ginecologia e obstetrícia (7), ortopedia e traumatologia (1), otorrinolaringologia (2).

\section{Avaliação sociodemográfica}

Um dos pesquisadores acompanhou o participante durante o preenchimento dos dados para solucionar possíveis dúvidas. Essa coleta de informaçóes aconteceu em local reservado, mantendo-se a privacidade dos participantes e em momento acordado com eles.

O formulário obteve informaçôes como gênero, idade, estado civil (casado ou não), renda familiar mensal 
(até 3 salários mínimos, entre 3 e 6 salários, ou acima de 6 salários) e carga horária de trabalho semanal (até 40 horas, entre 40 e 60 horas, ou acima de 60 horas) dos residentes.

\section{Avaliação do estresse e da síndrome de burnout}

A síndrome de burnout é caracterizada por três dimensôes: exaustão emocional, despersonalização e realização profissional reduzida. A exaustão emocional relaciona-se ao sentimento de esgotamento físico e mental, causado por uma intensa lida diária com problemas de outras pessoas; dessa forma, os profissionais sentem a energia emanada. A despersonalização, por sua vez, caracteriza-se pela dissimulação afetiva, desenvolvimento de atitudes negativas e ausência de sensibilidade, direcionadas aos receptores do serviço prestado. Por fim, a reduzida realização profissional é assinalada pela sensação de inutilidade, baixa autoestima, insatisfação com suas atividades e desmotivação, provocando, muitas vezes, a desistência profissional ${ }^{13}$.

$\mathrm{O}$ estudo da síndrome de burnout nos residentes aconteceu com a aplicaçáo do MBI (Maslach Burnout Inventory). O MBI é autoaplicado e avalia a forma com que o indivíduo vivencia o seu ambiente de trabalho. É solicitado que o entrevistado leia atentamente os itens e responda de acordo com a frequência de ocorrência do acontecimento, em uma escala de 0 a 6 . As questóes de 1 a 9 avaliam o nível de exaustão emocional; as de 10 a 17avaliam a realização profissional; e as de 18 a 22 relacionam-se à despersonalizaçáo ${ }^{14}$. Os pontos de corte utilizados para o diagnóstico de burnout são: para exaustão emocional: de 0 a 15 pontos (baixo), de 16 a 25 (médio) e de 26 a 54 (alto); para a despersonalização: de 0 a 2 (baixo), de 3 a 8 (médio) e de 9 a 30 (alto); e para a realização profissional: de 0 a 33 (baixo), de 34 a 42 (médio) e de 33 a 48 (alto). O diagnóstico de burnout é feito quando é constatado, a partir do MBI, que o indivíduo pontua nível alto em cansaço emocional ou despersonalização, ou nível baixo em realização pessoal ${ }^{15}$.

\section{Análise dos dados}

Todos os dados foram tabulados e analisados com uso do software Statistical Package for Social Sciences 22.0 (SPSS). A avaliação estatística da presença da síndrome de burnout determinada pelo MBI em relação a variáveis sociodemográficas foi realizada através dos testes exato de Fisher e Qui-quadrado $\left(\chi^{2}\right)$. A idade apresentou distribuição paramétrica pelo teste de normalidade Kolmogorov-Smirnov e foi analisada em relação à ocorrência da síndrome pelo teste t de Student. O nível de significância a fixado nos testes estatísticos foi de $5 \%(\mathrm{p}<0,05)$.

\section{Resultados}

A Tabela 1 mostra a distribuição dos dados sociodemográficos na amostra investigada. A idade dos participantes teve uma média de 29,2 $\pm 3,0$ anos, com mediana de 29, variando entre 24 e 35 anos. Em relação ao gênero e ao estado civil, percebe-se discreto predomínio de indivíduos do sexo masculino $(56,8 \%)$ e que não se encontravam casados no momento da pesquisa $(54,1 \%)$, respectivamente. Quanto à renda familiar mensal, notou-se que a maioria dos residentes apresentava renda acima dos $\mathrm{R} \$ 4.729,00(89,2 \%)$. No que diz respeito à carga horária, a maioria enquadrou-se nos grupos acima de 40 horas semanais $(89,2 \%)$. Os dados do MBI mostraram grande ocorrência da SB nos residentes avaliados nesta pesquisa $(75,7 \%)$.

Tabela 1: Distribuição dos dados sociodemográficos na amostra investigada

\begin{tabular}{|c|c|c|}
\hline Variáveis & $\mathbf{n}$ & $\%$ \\
\hline Idade (média \pm DP) & \multicolumn{2}{|c|}{$29,2 \pm 3,0$} \\
\hline \multicolumn{3}{|l|}{ Gênero } \\
\hline Feminino & 16 & 43,2 \\
\hline Masculino & 21 & 56,8 \\
\hline \multicolumn{3}{|l|}{ Estado civil } \\
\hline Não casado & 20 & 54,1 \\
\hline Casado & 17 & 45,9 \\
\hline \multicolumn{3}{|l|}{ Renda familiar mensal } \\
\hline Até R \$2.364,00 & 2 & 5,4 \\
\hline $\mathrm{R} \$ 2.365,00$ a $\mathrm{R} \$ 4.728,00$ & 2 & 5,4 \\
\hline Acima de $\mathrm{R} \$ 4.729,00$ & 33 & 89,2 \\
\hline \multicolumn{3}{|l|}{ Carga horária semanal } \\
\hline Até 40h & 4 & 10,8 \\
\hline $40-60 \mathrm{~h}$ & 18 & 48,7 \\
\hline $60-80 h$ & 15 & 40,5 \\
\hline \multicolumn{3}{|l|}{ Síndrome de burnout } \\
\hline Ausente & 9 & 24,3 \\
\hline Presente & 28 & 75,7 \\
\hline
\end{tabular}

A Tabela 2 apresenta a relação das variáveis sociodemográficas com a ocorrência de SB. Apesar de algumas tendências como redução proporcional da ocorrência de $\mathrm{SB}$ em indivíduos casados, com menor renda familiar mensal e com menores cargas horárias de trabalho serem observadas, nenhuma relação estatisticamente significativa foi identificada nessa análise. 
Tabela 2: Relação das variáveis sociodemográficas com a ocorrência de SB

\begin{tabular}{lccc} 
& \multicolumn{3}{c}{ Síndrome de burnout } \\
Variáveis & Ausente & Presente & p \\
Idade (média \pm DP)* & $28,3 \pm 2,2$ & $29,5 \pm 3,1$ & 0,295 \\
Gênero** & & & \\
Feminino & $4(25,0 \%)$ & $12(75,0 \%)$ & 0,118 \\
Masculino & $5(23,8 \%)$ & $16(76,2 \%)$ & \\
Estado civil** & & & \\
Não casado & $3(15,0 \%)$ & $17(85,0 \%)$ & 0,251 \\
Casado & $6(35,3 \%)$ & $11(64,7 \%)$ & \\
Renda familiar mensal** & & & \\
Até R $\$ 2.364,00$ & $1(50,0 \%)$ & $1(50,0 \%)$ & 0,223 \\
R\$2.365,00 a R $\$ 4.728,00$ & $1(50,0 \%)$ & $1(50,0 \%)$ & \\
Acima de R $\$ 4.729,00$ & $7(21,2 \%)$ & $26(78,8 \%)$ & \\
Carga horária semanal** & & & \\
Até 40h & $1(25,0 \%)$ & $3(75,0 \%)$ & 0,874 \\
40-60h & $5(27,8 \%)$ & $13(72,2 \%)$ & \\
60-80h & $3(20,0 \%)$ & $12(80,0 \%)$ & \\
\hline
\end{tabular}

$\mathrm{DP}=$ Desvio padrão.

* Teste t de Student.

**Teste Qui-quadrado $\left(\chi^{2}\right)$.

\section{Discussão}

As características da síndrome de burnout são despersonalização, desgaste emocional e diminuição da realizaçáo profissional do indivíduo ${ }^{16}$. É uma condição comum em profissóes que demandam grande cuidado e atenção para com o outro, como assistentes sociais, professores e trabalhadores da área da saúde. Profissionais da área de saúde, principalmente os médicos, são expostos diariamente a altos níveis de pressão e estresse. A tensão persistente pode levar à exaustão e a danos físicos ou psicológicos ${ }^{17}$.

Em estudo feito por Trindade e Lautert em 2010, foram entrevistados 86 trabalhadores de unidades básicas de saúde da cidade de Santa Maria, com a utilização do MBI para avaliar a prevalência de burnout. De acordo com tal estudo, a prevalência dessa síndrome é maior em trabalhadores jovens, sendo mais comum até os 30 anos de idade. Isso pode ser atribuído à insegurança causada pela pouca experiência do profissional. Muitas vezes, existe idealização associada a expectativas elevadas, que, muitas vezes, náo se concretizam. Essa excessiva motivação, paradoxalmente, torna esses indivíduos mais vulneráveis à $\mathrm{SB}^{16}$. Neste estudo, não se observou relação entre a idade e a ocorrência da $\mathrm{SB}$, o que pode ter pertinência com a baixa amplitude na faixa etária verificada nesta pesquisa ( 24 a 35 anos), não permitindo, assim, evidenciar associação direta entre a idade e a presença de $\mathrm{SB}$ em residentes médicos.
A SB em residentes detectada neste estudo não revelou diferenças significativas da presença dessa síndrome entre os gêneros, o que também foi evidenciado em trabalho de Lima et al. ${ }^{18}$ com 133 médicos residentes do Hospital de Clínicas da Universidade Federal de Uberlândia. Observa-se que ambos os sexos não possuem margens estatísticas destoantes e, portanto, acredita-se que os residentes compartilham fatores estressores semelhantes que condicionam o desenvolvimento da doença.

Gabbe et al. ${ }^{19}$, em estudo com médicos obstetras e ginecologistas, constataram menor prevalência de burnout para os médicos que tinham um cônjuge. Isso, possivelmente, deve-se ao fato de que os médicos casados contam com um(a) parceiro(a) para dividir as tarefas cotidianas e responsabilidades financeiras, bem como para apoiá-los em momentos de estresse e demais adversidades. No entanto, também não se identificou relação estatística entre a SB e o estado civil dos residentes médicos investigados neste trabalho. Indivíduos não casados não necessariamente representam pessoas solteiras ou sem amparo familiar. Essa característica em nossa investigação também pode ter afetado a interpretação estatística dos dados, não permitindo a identificação de inferências que determinem as associaçóes entre estado civil e SB.

A renda familiar também não teve relação significativa com a ocorrência de SB na amostra estudada. Por outro lado, estudo feito por Sobrinho et al. ${ }^{20} \mathrm{em}$ médicos de UTI mostrou que a SB foi mais comum naqueles que referiram renda mensal inferior a $\mathrm{R} \$ 5.000,00$. É provável que a renda não esteja relacionada à $\mathrm{SB}$ nesta pesquisa devido ao fato de a maioria dos médicos residentes avaliados se enquadrar na categoria que recebe mais do que $\mathrm{R} \$ 4.729,00$, sendo que apenas quatro participantes não alcançavam essa renda. Tal discrepância na distribuiçấo dos casos entre as categorias salariais pode ter interferido na identificação de associações entre a renda familiar mensal e a ocorrência da SB. Outro detalhe é o baixo valor amostral investigado neste estudo, o que pode atrapalhar a identificação de algumas associações estatísticas como a renda.

A carga horária de trabalho semanal também não se mostrou relacionada aos casos com SB nesta pesquisa. Esse resultado, no entanto, também diverge do estudo feito com 297 médicos plantonistas, no qual Sobrinho et al. ${ }^{20}$ demonstraram maior prevalência de $\mathrm{SB}$ em médicos que trabalhavam mais de 12 horas no final de semana. Acredita-se que, em alguns casos, a baixa remuneração fornecida pelas instituiçôes não é suficiente para suprir as despesas familiares dos profissionais médicos; assim, estes se submetem a cargas horárias extras e exaustivas, em regime de plantão, a fim de compensar a renda insuficiente. $\mathrm{O}$ excesso de carga de trabalho, portanto, poderia determinar exigências 
de trabalho excedentes às capacidades, às competências e aos conhecimentos do trabalhador, afetando a qualidade do serviço oferecido ${ }^{21}$. Em estudo realizado por Satterfield et al. ${ }^{22}$ com residentes médicos, realizou-se uma avaliação durante todo o período de residência para determinar as diferentes emoçóes que estes sofrem nesse período, que apontou excesso de cargas de trabalho, privação de sono e preocupação com erros médicos como fatores que levam à perda de perspectiva profissional, com diminuiçấo do cuidado com pacientes e colegas de trabalho, gerando impactos negativos nas relaçóes e no desempenho. Dessa forma, como evidenciado na avaliaçáo de renda familiar, muitos residentes, apresentaram-se nas categorias mais altas de renda familiar, o que pode ter colaborado para minimizar o efeito das altas cargas de trabalho vivenciadas por grande parte dos casos deste estudo.

Apesar de a ausência de relaçóes estatísticas, este trabalho evidenciou uma grande ocorrência de $\mathrm{SB}$ em residentes médicos de Montes Claros/MG (75,7\%). O estresse, que gera a síndrome de burnout é cada vez mais presente entre os residentes, e a magnitude desse problema é resultante da interação de três vertentes: profissional, situacional e pessoal. Ao se sentirem exaustos, os profissionais relatam um sentimento de sobrecarga física e emocional acompanhada de dificuldade para relaxar, relatando um estado de fadiga diário. Uma vez exaustos, existe uma diminuição dos recursos internos e da energia para enfrentar as situaçóes vivenciadas no trabalho $^{18}$. Esse estresse ocupacional costuma levar à desestruturação no ambiente de trabalho e no círculo social como um todo, por isso é importante que haja perspectivas de enfrentamento e superação dos diversos fatores estressores, assim como a prevenção e a promoçâo da qualidade de vida dos residentes ${ }^{23}$.

A profissão médica é uma atividade que lida com as situações mais temidas pelo ser humano: a doença, o sofrimento, o desamparo e a morte. Dessa forma, o profissional terá de lidar com as tensóes emocionais de outras pessoas em momentos em que estas se encontram fragilizadas, com a expectativa de cura dos pacientes, que nem sempre é possível, com as limitaçóes impostas a ele pela capacidade própria ou pelo sistema de saúde, com a carga horária extenuante, entre outros. Tudo isso leva o indivíduo a uma situação desconfortável e ao sentimento de desamparo ${ }^{24}$. O maior problema social relacionado ao burnout em médicos é a possibilidade de diminuição do envolvimento e da dedicação necessários ao atendimento dos pacientes, culminando ainda em uma menor realizaçáo profissional ${ }^{18}$.

Portanto, a perda da qualidade de vida dos profissionais médicos causada pela SB pode acarretar reduçáo da qualidade no atendimento médico e, consequentemente, afetar de forma indireta a saúde da população. A melhora desses índices pode ser alcançada através de modificaçôes das estruturas pedagógicas dos cursos de residência médica, que possibilitariam redução da carga horária semanal e aumento do valor da bolsa, diminuindo, assim, a necessidade de plantóes extras, bem como permitindo realização de exercícios físicos durante, pelo menos, 10 minutos ao dia e de terapias para controle do estresse ${ }^{17}$.

Neste estudo, deve-se, no entanto, fazer uma ressalva com relação ao baixo número de residentes avaliados, o que prejudica a análise estatística que busca relaçóes entre alguns possíveis determinantes de risco investigados no trabalho. Porém, a identificação de grande proporção de indivíduos afetados com a SB serve como alerta acerca das metodologias aplicadas nos cursos de residência médica ou mesmo para os profissionais que estão atuando nestes.

\section{Conclusão}

A alta incidência de síndrome de burnouté uma realidade entre estudantes de residências médicas, o que torna imperativa a busca de medidas intervencionistas e curativas para diminuir o alto desgaste a que estáo expostos os profissionais dessa área. Este trabalho com 37 residentes de três hospitais de Montes Claros/MG mostra alto índice $(75,7 \%)$ de burnout, embora não tenha ficado evidente sua relação com gênero, estado civil, renda familiar ou carga horária. A despeito disso, que pode ter relação com quantidade de residentes avaliados, os dados reforçam a importância de medidas de prevenção do desgaste físico e psíquico desses profissionais. A importância de estudos como este está na tentativa de compreender o atual processo de desenvolvimento de burnout e suas complicaçóes entre os médicos residentes. Destaca-se a necessidade de melhorar as condiçóes dos serviços de residência médica no país e desenvolver práticas de apoio aos residentes, no intuito de minimizar os problemas de burnout e permitir que essa importante etapa de complementação da formação acadêmica seja pautada por excelência no aprendizado e satisfação pessoal e profissional.

\section{Conflito De Interesses}

Os autores não declaram conflitos de interesse.

\section{Referências}

1. Nogueira-Martins LA, Jorge MR. Natureza e magnitude do estresse na residência médica. Rev Assoc Med Bras. 1998;44(1):28-34. 
2. Conselho Federal de Medicina. Resolução CFM no 1973. Dispóe sobre a nova redação do Anexo II da Resolução CFM no $1.845 / 08$, que celebra o convênio firmado entre o Conselho Federal de Medicina (CFM), a Associação Médica Brasileira (AMB) e a Comissão Nacional de Residência Médica (CNRM) [Internet]. Brasília, DF; 1 ago 2011. [citado em 2017jul23]. Disponível em: https://goo.gl/oYJac9

3. Costa EFO, Santana YS, Santos ATRA, Nogueira-Martins LA, Melo EV, Andrade TM. Sintomas depressivos entre internos de medicina em uma universidade pública brasileira. Rev Assoc Med Bras. 2012;58(1):53-9.

4. Maslach C, Jackson SE. The role of sex and family variables in burnout. Sex Roles. 1985;12(7-8):837-51.

5. Gonçalves TB, Leitão AKR, Botelho BS, Marques RACC, Hosoume VSN, Neder PRB. Prevalência de síndrome de burnout em professores médicos de uma universidade pública em Belém do Pará. Rev. Bras. Med. Trab. 2011;9(2):85-9.

6. Hyeda A, Handar Z. Avaliação da produtividade na síndrome de burnout. Rev Bras Med Trab. 2011;9(2):78-84.

7. Fahrenkopf AM, Sectish TC, Barger LK, Sharek PJ, Lewin $\mathrm{D}$, Chiang VW, et al. Rates of medication errors among depressed and burnt out residents: prospective cohort study. BMJ. 2008;336(7642):488-91.

8. Fabichak C, Silva-Júnior JS, Morrone LC. Síndrome de burnout em médicos residentes e preditores organizacionais do trabalho. Rev. Bras. Med. Trab. 2014;12(1):79-84.

9. Maslach C, Jackson SE. The measurement of experienced burnout. J Organ Behav. 1981;2(2)99-113.

10. Freudenberger HJ. The staff burn-out syndrome in alternative institutions. Psychol Psychother. 1975;12(1):73-82.

11. Kahill S. Symptoms of professional burnout: a review of the empirical evidence. Can Psychol. 1988;29(3):284-97.

12. van der Ploeg HM, van Leeuwen JJ, Kwee MGT. Burnout among dutch psychotherapists. Psychol Rep. 1990;67(1):107-12.

13. Ferrari R, França FM, Magalhães J. Avaliação da síndrome de burnout em profissionais de saúde: uma revisão integrativa da literatura. Gestão e Saúde. 2012;3(3):1150-65.
14. Cracco CLAC, Salvador JA. Identificação da síndrome de burnout na equipe de enfermagem de uma unidade de pronto atendimento [trabalho de conclusão de curso]. Lins: Unisalesiano; 2010.

15. Grunfeld E, Whelan TJ, Zitzelsberger L, Willan AR, Montesanto B, Evans WK. Cancer care workers in Ontario: prevalence of burnout, job stress and job satisfaction. CMAJ. 2000;(2):166-9.

16. Trindade LL, Lautert L. Síndrome de burnout entre os trabalhadores da Estratégia de Saúde da Família. Rev Esc Enferm USP. 2010;44(2):274-79.

17. Romani M, Ashkar K. Burnout among physicians. Libyan J Med. 2014;9(1):23556.

18. Lima FD, Buunk AP, Araújo MBJ, Chaves JGM, Muniz DLO, Queiroz LB. Síndrome de burnout em residentes da Universidade Federal de Uberlândia - 2004. Rev Bras Educ Med. 2007;31(2):137-46.

19. Gabbe SG, Melville J, Mandel L, Walker E. Burnout in chairs of obstetrics and gynecology: diagnosis, treatment and prevention. Am J Obstet Gynecol. 2002;186(4):601-12.

20. Sobrinho CLN, Barros DS, Tironi MOS, Marques Filho ES. Médicos de UTI: prevalência da síndrome de burnout, características sociodemográficas e condiçôes de trabalho. Rev Bras Educ Med.2010;34(1):106-15.

21. Cumbe VFJ. Síndrome de burnout em médicos e enfermeiros cuidadores de pacientes com doenças neoplásicas em serviços de Oncologia [dissertação]. Porto: Universidade do Porto; 2010.

22. Satterfield JM, Becerra C. Developmental challenges, stressors and coping strategies in medical residents: a qualitative analysis of support groups. Med Educ.2010;44(9):908-16.

23. Silva DP, Silva MNRMO. O trabalhador com estresse e intervençốes para o cuidado em saúde. Trab. educ. saúde. 2015;13(1):201-14.

24. Katsurayama M, Gomes NM, Becker MAD, Santos MC, Makimoto FH, Santana LLO. Avaliação dos níveis de estresse psicológico em médicos residentes e não residentes de hospitais universitários. Psicol. hosp.2011;9(1):75-96.

\section{Como citar este artigo:}

Ferreira ECN, Ribeiro FV, Santos Neto RP, Santos BN, Espirito Santo LR, Prince KA, Oliveira MVM. Síndrome de burnout em residentes médicos de Montes Claros/MG. Rev. Aten. Saúde. 2017;15(53):44-49. 